\title{
Protein micro- and nano-patterning using aminosilanes with protein-resistant photolabile protecting groups.
}

\begin{abstract}
An approach to the integration of nanolithography with synthetic chemical methodology is described, in which near-field optical techniques are used to selectively deprotect films formed by the adsorption of aminosilanes protected by modified 2nitrophenylethoxycarbonyl (NPEOC) groups. The NPEOC groups are functionalized at the $\mathrm{m}$ - or p-position with either a tetraethyleneglycol or a heptaethylene glycol adduct. We describe the synthesis of these bioresistant aminosilanes and the characterization of the resulting photoreactive films. Photodeprotection by exposure to UV light $(\lambda=325 \mathrm{~nm})$ yielded the amine with high efficiency, at a similar rate for all four adsorbates, and was complete after an exposure of $2.24 \mathrm{~J} \mathrm{~cm}-2$. Following photodeprotection, derivatization by trifluoroacetic anhydride was carried out with high efficiency. Micropatterned samples, formed using a mask, were derivatized with aldehyde-functionalized polymer nanoparticles and, following derivatization with biotin, were used to form patterns of avidin-coated polymer particles. Fluorescence microscopy and atomic force microscopy data demonstrated that the intact protecting groups conferred excellent resistance to nonspecific adsorption. Nanometer-scale patterns were created using scanning near-field photolithography and were derivatized with biotin. Subsequent conjugation with avidin-functionalized polymer nanoparticles yielded clear fluorescence images that indicated dense attachment to the nanostructures and excellent protein resistance on the surrounding surface. These simple photocleavable protecting group strategies, combined with the use of near-field exposure, offer excellent prospects for the control of surface reactivity at nanometer resolution in biological systems and offer promise for integrating the top-down and bottom-up molecular fabrication paradigms.
\end{abstract}

Keyword: SNP; Protecting groups; Aminosilanes; Protein-resistant photolabile. 